\title{
Di-electron production from vector mesons with medium modifications in heavy ion collisions
}

\author{
Hao-jie Xu, ${ }^{1}$ Hong-fang Chen, ${ }^{1}$ Xin Dong, ${ }^{2}$ Qun Wang, ${ }^{1}$ and Yi-fei Zhang ${ }^{1}$ \\ ${ }^{1}$ Department of Modern Physics, University of Science and Technology of China, Anhui 230026, People's Republic of China \\ ${ }^{2}$ Nuclear Science Division, Lawrence Berkeley National Laboratory, Berkeley, California 94720, USA
}

\begin{abstract}
We reproduce the di-electron spectra in the low and intermediate mass regions in most central $\mathrm{Au}+\mathrm{Au}$ collisions by the STAR Collaboration incorporation of the STAR detector acceptance. We also compare our results with PHENIX data constrained by the PHENIX acceptance. We include the medium modifications of vector mesons from scatterings of vector mesons by mesons and baryons in the thermal medium. The freezeout contributions from vector mesons are also taken into account. The space-time evolution is described by a $2+1$ dimensional ideal hydrodynamic model. The backgrounds from semi-leptonic decays of charm hadrons are simulated by the PYTHIA event generator and corrected by the nuclear modification factor of electrons from charm decays. It is difficult to extract the thermal contributions from those from charm decays in the invariant mass spectra alone and in the current detector acceptances. Other observables such as transverse momenta and collective flows may provide additional tools to tag these sources.
\end{abstract}

\section{INTRODUCTION}

The electromagnetic probes such as photons and dileptons are expected to provide clean signatures for the quark gluon plasma (QGP) in heavy ion collisions due to their instant emissions once produced [1-7]. These thermal photons and dileptons contain undistorted information about the space-time trace of the new state of matter formed in such collisions. The invariant mass spectrum is usually divided into the low, intermediate and high mass regions (LMR, IMR and HMR), based on the notion that each region is dominated by different sources of dileptons. In the LMR, $M \lesssim 1 \mathrm{GeV}$, dileptons are mainly from vector meson decays and may be related to chiral symmetry restoration [8 13]. In the HMR, $M \gtrsim 3 \mathrm{GeV}$, dileptons are dominated by the Drell-Yan process and quarkonium decays. In the IMR, $1 \lesssim M \lesssim 3 \mathrm{GeV}$, it was argued that dileptons from semi-leptonic decays of correlated open charm hadrons are dominant [14].

The medium modifications of the $\rho$ meson spectral functions are successful in describing the di-muon enhancement in the LMR of the NA60 experiment at the SPS energy [11, 12, 15]. The PHENIX and STAR collaborations also observed such an enhancement in the dielectron spectra at the RHIC energy [16, 17]. The thermal quark-antiquark annihilation in the QGP phase is expected to give a measurable signal in the IMR for the deconfinement phase transition at RHIC energy [18]. However, in this mass region, the di-lepton yield from semileptonic decays of open charm mesons increases rapidly with the collisional energy. The single leptons from open charm mesons and their dynamic correlations are expected to undergo medium modifications. The question is: to what extent the di-leptons from charm hadrons with medium modifications mix up with the thermal contributions from the QGP in the IMR. Another issue is that the dilepton spectra measured by the STAR and PHENIX collaborations are very different in the LMR. It is worthwhile to to look at this disagreement closely by using the Monte Carlo simulation incorporating the different acceptances of STAR and PHENIX detectors.

In this paper, we try to reproduce the data of dielectron invariant mass spectra in the LMR and IMR in central $\mathrm{Au}+\mathrm{Au}$ collisions at $200 \mathrm{GeV}$. We will include the medium modifications of the vector mesons and charm hadrons. The acceptances of STAR and PHENIX detectors are incorporated in our calculation. We will use a $2+1$ dimension ideal hydrodynamic model to give the space-time evolution of the fireball, where the parameters are determined by fitting the data of transverse momenta of long life hadrons (pions, Kaons and protons). The spectra of charm hadrons $\left(D^{0}, D^{ \pm}, D_{s}\right.$ and $\left.\Lambda_{c}\right)$ are given by a simulation of the PYTHIA event generator. We neglect the Dalitz decay channel for $\pi^{0}: \pi^{0} \rightarrow e^{+} e^{-} \gamma$ but include those for $\eta$ and $\omega: \eta \rightarrow e^{+} e^{-} \gamma$ and $\omega \rightarrow e^{+} e^{-} \pi^{0}$. The contribution from pion's Dalitz decay is mainly below $m_{\pi}$ and irrelevant to our current range of the invariant mass.

\section{PARAMETERS IN HYDRODYNAMIC MODEL}

We use a $2+1$ dimensional ideal hydrodynamic model [18] to give the space-time evolution of the medium created in heavy ion collisions. We choose two types of the Equation of state (EOS) [19 22], S95P-CE (CE) with complete chemical equilibrium to very low temperatures and a wide range of phase transition temperatures from $184 \mathrm{MeV}$ to $220 \mathrm{MeV}$, and S95P-PCE (PCE) with partial chemical equilibrium below chemical freezeout temperature $T_{\text {chem }}=165 \mathrm{MeV}$ [43]. After kinetic freeze-out, we use the Cooper-Frye formula [23, 24] to obtain the momentum spectra for each hadron species

$$
E \frac{d N_{i}}{d^{3} p}=\frac{d N_{i}}{d y p_{T} d p_{T} d \phi}=\frac{g_{i}}{(2 \pi)^{3}} \int_{T_{f}} d \Sigma_{\mu} p^{\mu} n_{i}(x, u \cdot p),
$$




\begin{tabular}{|c|c|c|c|c|}
\hline set & EoS & $T_{\text {chem }}[\mathrm{MeV}]$ & $T_{f}[\mathrm{MeV}]$ & $e_{f}\left[\mathrm{GeV} / \mathrm{fm}^{3}\right]$ \\
\hline \hline S1 & S95P-CE & - & 136 & 0.12 \\
\hline S2 & S95P-PCE & 165 & 136 & 0.275 \\
\hline S3 & S95P-PCE & 165 & 106 & 0.12 \\
\hline
\end{tabular}

Table I: Parameter sets. We choose most central collisions with $b=2.4 \mathrm{fm}$. The initial conditions are chosen to be: $\tau_{0}=0.4 \mathrm{fm}, e_{0}=45 \mathrm{GeV} / \mathrm{fm}^{3}\left(T_{0}=395 \mathrm{MeV}\right)$.

where $\Sigma_{\mu}$ denotes the normal vector of the freezeout hypersurface, $T_{f}$ is the kinetic freeze-out temperature on the freezeout hypersurface, $n_{i}$ is the phase space distribution function for the baryon/meson species $i$ which can be Fermi-Dirac/Bose-Einstein distribution and $g_{i}$ is its degeneracy factor, and $p_{T}$ denotes the transverse momentum.

Beside the EOS, there are some free parameters which should be fixed in the hydrodynamical model, such as equilibration time $\tau_{0}$, the initial energy density $e_{0}$ (or initial temperature $T_{0}$ ) and the kinetic freeze-out energy density $e_{f}\left(\right.$ or $T_{f}$ ). At the RHIC energy $\sqrt{s_{N N}}=200$ $\mathrm{GeV}$ for $\mathrm{Au}+\mathrm{Au}$ collisions [25, 26], we constrain these parameters with STAR and PHENIX data for the rapidity densities of multiplicities, $d N_{i} / d y$, and the $p_{T}$ spectra for long-life hadrons (pions, koans and protons). The distribution of the initial energy density is determined by the Glauber model with $5 \%$ of the contribution from binary collisions. We focus on most central collisions with impact parameter $b=2.4 \mathrm{fm}$ throughout the paper and assume a system with vanishing net baryon number.

To make a comparison, we choose the same initial conditions for two $\operatorname{EOS~}\left(\tau_{0}=0.4 \mathrm{fm}, e_{0}=45 \mathrm{GeV} / \mathrm{fm}^{3}\right.$ (or $\left.T_{0}=395 \mathrm{MeV}\right)$ ), but freeze-out conditions are different due to different relations between the energy density and temperature. The same initial conditions imply that the entropy densities are chosen to be the same for these two EOS. We choose $T_{f}=136 \mathrm{MeV}\left(e_{f}=0.12 \mathrm{GeV} / \mathrm{fm}^{3}\right)$ for $\mathrm{CE}$ and roughly reproduce the $p_{T}$ spectra for the pions and kaons, as shown in Fig. (1)(a)). But the proton yield is under-estimated. For PCE, we choose the kinetic freeze-out occurs at either the same energy density or the same temperature as CE, i.e. $\left(e_{f}, T_{f}\right)=\left(0.12 \mathrm{GeV} / \mathrm{fm}^{3}\right.$, $106 \mathrm{MeV})$ or $\left(0.275 \mathrm{GeV} / \mathrm{fm}^{3}, 136 \mathrm{MeV}\right)$. The $p_{T}$ spectra with PCE are shown in Fig. (1(b)). The parameter sets are listed in Tab. (I).

The PCE scenario has a feature compared to the $\mathrm{CE}$ one [19]: $d N_{i} / d y$ and $p_{T}$ spectra in the low $p_{T}$ range $\left(p_{T}<1 \mathrm{GeV}\right)$ are almost independent of $T_{f}$. However, the high $T_{f}$ will reduce the dilepton production rate by shortening the evolution time. So we can regard $T_{f}$ as a tuning parameter to the dilepton production rate from the in-medium vector meson decay.

We give a few comments about the yields of vector mesons, whose multiplicity ratios, $\rho / \pi, \omega / \pi$ and $\phi / \pi$, are given in Tab. (III). Most of the decays of the $\omega$ and $\phi$ mesons take place after the kinetic freeze-out. The yields of $\omega$ and $\phi$ are in good agreement with the data and will
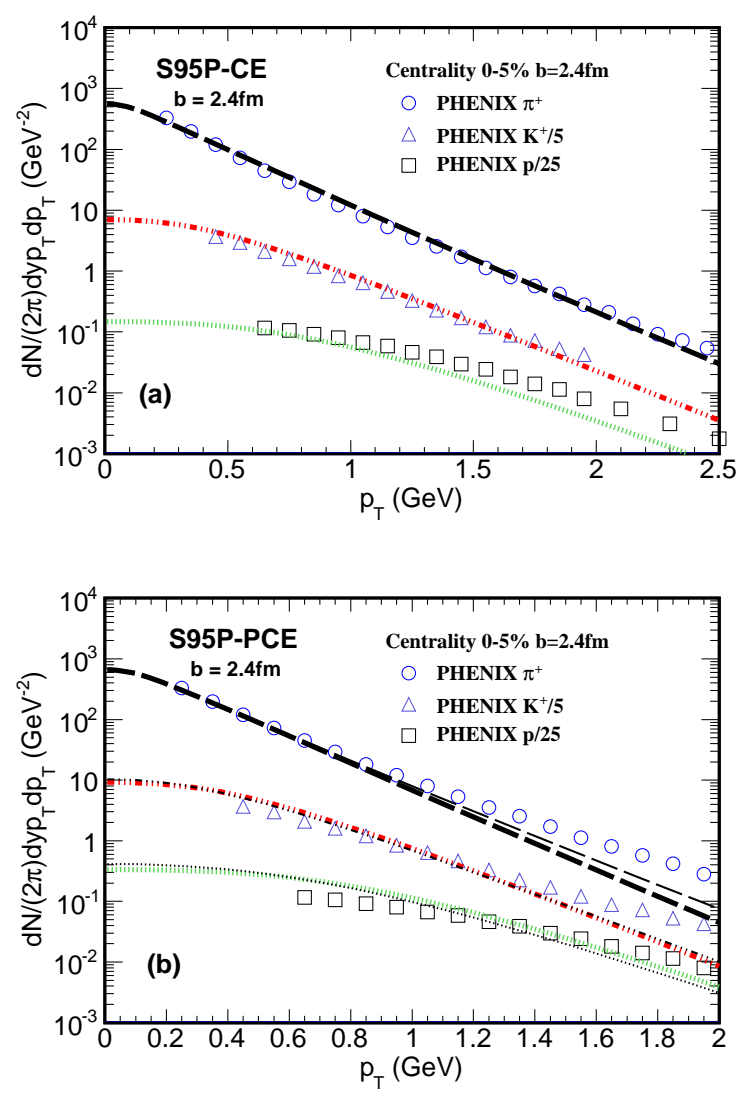

Figure 1: (Color online) Transverse momentum spectra for $\pi^{+}$ (black-dashed), $K^{+}$(red-dash-dot-dot-dotted) and $p$ (greendotted) for central collisions with centrality 0-5\%. The data are from the PHENIX collaboration [26]. (a) S95P-CE and (b) S95P-PCE EOS are used. In (b), the differences between $T_{f}=106 \mathrm{MeV}$ (thick lines) and $T_{f}=136 \mathrm{MeV}$ (thin lines) in the low $p_{T}$ range are small.

contribute to the dilepton rate. We see that the ratio $\rho / \pi$ at the freeze-out is smaller than the data, but we note that most of the $\rho$ mesons decay in the thermal medium earlier than the kinetic freeze-out whose contribution to the dilepton production dominates the dilepton spectra. Hereafter we will use the parameter set S3 of PCE as a default choice unless stated explictly. The comparison will be made with other parameter sets.

\section{DILEPTON EMISSIONS IN HEAVY ION COLLISIONS}

In the thermalized medium, hadron gas (HG) or quark gluon plasma (QGP), the dilepton production rate per 


\begin{tabular}{|c|c|c|c|c|c|}
\hline sets & $\pi^{+}$ & $p$ & $\rho / \pi$ & $\omega / \pi$ & $\phi / \pi$ \\
\hline \hline S1 & 285.7 & 12.2 & $7.91 \times 10^{-2}$ & $7.49 \times 10^{-2}$ & $1.86 \times 10^{-2}$ \\
\hline S2 & 270.2 & 23.6 & $7.78 \times 10^{-2}$ & $9.85 \times 10^{-2}$ & $2.89 \times 10^{-2}$ \\
\hline S3 & 261.7 & 24.6 & $5.50 \times 10^{-2}$ & $9.82 \times 10^{-2}$ & $3.13 \times 10^{-2}$ \\
\hline PHENIX & 281.8 & 18.4 & $1.03 \times 10^{-1}$ & $8.98 \times 10^{-2}$ & $2.14 \times 10^{-2}$ \\
\hline STAR & 327 & 34.7 & $1.69 \times 10^{-1}$ & - & $2.65 \times 10^{-2}$ \\
\hline
\end{tabular}

Table II: Rapidity densities $d N_{i} / d y$ for meson/proton yields in most central collisions with $b=2.4 \mathrm{fm}$. The PHENIX data are taken from Ref. [16, 26], where they only have the $\pi^{+}$ data in most central collisions and their $\rho$ data are from the fragmentation model. The STAR data are from Ref. [27, 28].

unit volume is given by

$$
\begin{aligned}
\frac{d N_{l l}}{d^{4} x d^{4} p} & =-\frac{\alpha}{4 \pi^{4}} \frac{1}{M^{2}} n_{B}(p \cdot u)\left(1+\frac{2 m_{l}^{2}}{M^{2}}\right) \\
& \times \sqrt{1-\frac{4 m_{l}^{2}}{M^{2}}} \operatorname{Im}^{R}(p, T) .
\end{aligned}
$$

Here $m_{l}$ is the lepton mass, $\alpha=e^{2} / 4 \pi$ is the fine structure constant with the electric charge $e$ for leptons, $p=\left(p_{0}, \mathbf{p}\right)=p_{1}+p_{2}$ is the dilepton 4-momentum and $M=\sqrt{p^{2}}, n_{B}=1 /\left(e^{p \cdot u / T}-1\right)(T$ and $u$ are the local temperature and fluid velocity respectively) is the Bose distribution function, $\Pi_{\mu \nu}^{R}$ is the retarded photon polarization tensor from the quark or hadronic loop, and $\Pi^{R}=\frac{1}{3} \Pi_{\mu}^{R \mu}$. For the partonic phase, $\Pi^{R}$ given by the Born term reflects the lowest order process $q \bar{q} \rightarrow \gamma^{*} \rightarrow l^{+} l^{-}$. For the hadronic phase, $\Pi^{R}$ is further related to the retarded vector-meson propagator $D_{V}^{R}$ with $V=\rho, \omega, \phi$ via $\operatorname{Im} \Pi^{R}=-\left(e^{2} m_{V}^{4} / g_{V}^{2}\right) \operatorname{Im} D_{V}^{R}$, where $g_{V}$ is the photon-vector-meson coupling constant in the vector meson dominance model, and $m_{V}$ is the vector-meson mass. The retarded vector meson propagator is

$$
\operatorname{Im} D_{V}^{R}=\frac{\operatorname{Im} \Pi_{V}^{R}}{\left(p^{2}-m_{V}^{2}+\operatorname{Re} \Pi_{V}^{R}\right)^{2}+\left(\operatorname{Im} \Pi_{V}^{R}\right)^{2}}
$$

where $\Pi_{R}$ is the contraction of the retarded vector meson polarization tensor.

We use the hadronic many body effective theory [29] to calculate in-medium $\rho$ spectral functions by scattering with surrounding mesons. Though we assume a net baryon free system at RHIC energy, the effective chemical potentials in the $\mathrm{PCE}$ scenario will give a considerable number of baryons [30], and we assume that there are equal number of anti-baryons which give the same contribution as the baryons to the $\rho$ spectral functions. To include the baryonic (including anti-baryonic) contributions, we use the empirical scattering amplitude method [31] which agrees with the hadronic many body effective theory [32]. Here we only consider the coupling of the $\rho$ meson with baryonic resonances in the medium and set the momentum $q=300 \mathrm{MeV}$ for the $\rho$ meson in-medium propagator. The in-medium $\rho$ meson spectral functions with and without baryonic contributions are shown in

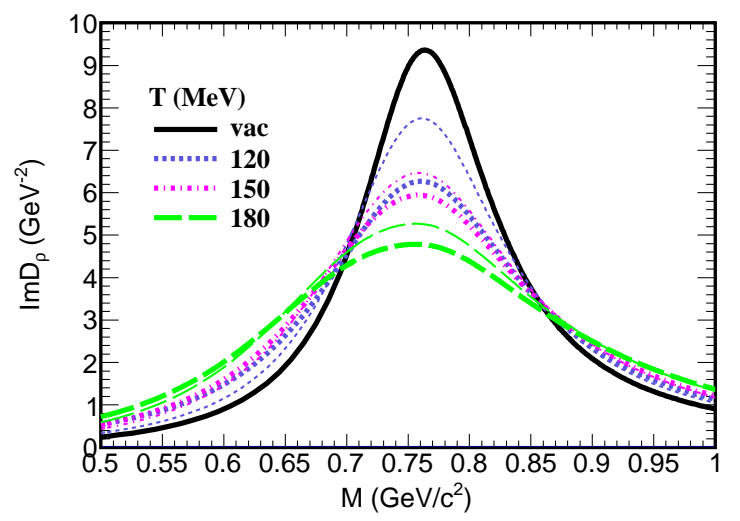

Figure 2: The imaginary parts of the in-medium $\rho$ meson propagators (or in-medium spectral functions) with (thick lines) and without (thin lines) baryonic contributions. The chemical potentials in PCE EOS are used.

Fig. (2) at different temperatures but at a fixed momentum $q=300 \mathrm{MeV}$. The differences between with and without baryonic contributions are larger at low temperatures than at high temperatures.

Since the collision rate in a meson gas around the transition temperature indicates a large broadening of $\phi$ meson spectra due to binary collisions [33], we include this effect via a schematic estimate as follows (with $T_{0}=150$ $\mathrm{MeV})$,

$$
\Gamma_{\phi_{\text {coll }}} \simeq(22 \mathrm{MeV})\left(\frac{T}{T_{0}}\right)^{6} .
$$

In Fig. (3) (a)) we show the invariant mass spectra of thermal di-electrons at the RHIC energy $200 \mathrm{GeV}$ for most central $\mathrm{Au}+\mathrm{Au}$ collisions. Beside the $\rho$ component (red-dotted line) in the hadronic phase in an early study [18], we include the in-medium $\omega$ (magenta-shortdashed line) and $\phi$ (light-brown-dash-dotted line) contributions to thermal di-electrons. The thermal di-electrons are dominated by the in-medium $\rho$ mesons, while the $\omega$ contribution is submerged under the broadened $\rho$ spectra. The thermal spectra with the CE EOS (green-dashdotted-dotted line) has also been shown. The production rate in the PCE scenario is larger in the invariant mass range below free $\rho$ mass than in the $\mathrm{CE}$ one, though the temperatures with PCE are lower. This is because the chemical potentials in the PCE scenario lead to a larger broadening of the $\rho$ spectral function and an enhancement factor $e^{2 \mu_{\pi} / T}$ compared with the $\mathrm{CE}$ scenario. The enhancement at low masses in the PCE scenario is more obvious at lower $T_{f}$. We will come back to this issue later. These two EOS have the same partonic contributions because their differences only occur in the hadronic phase.

The dilepton emission rate from the freezeout vector 

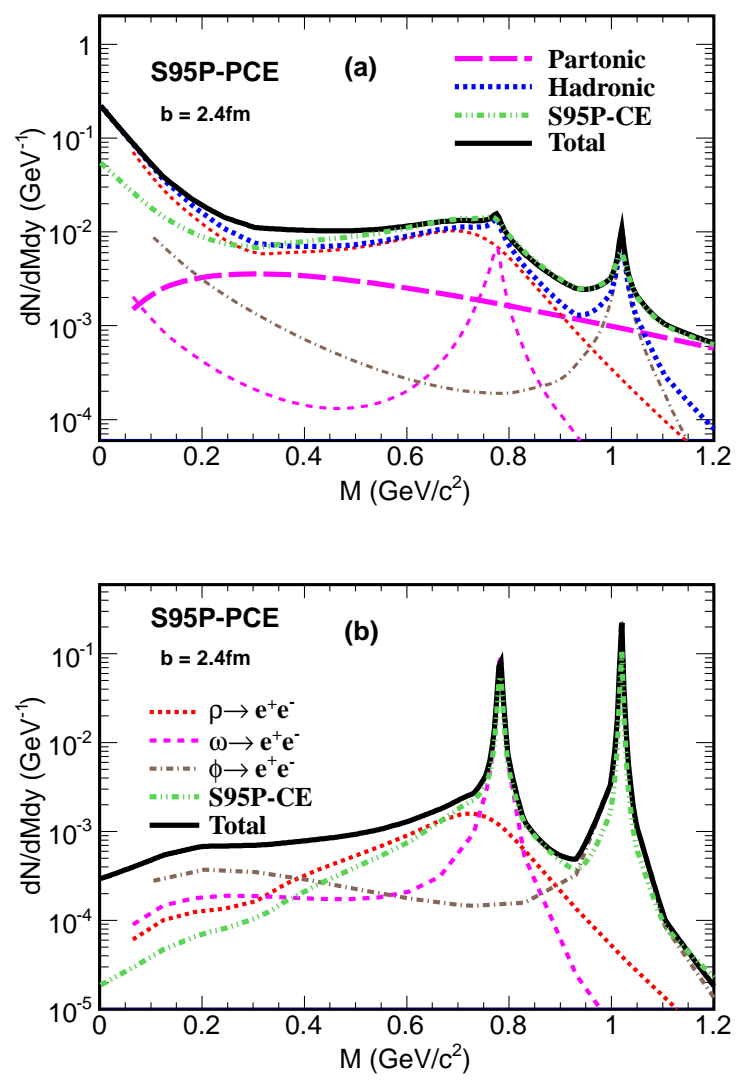

Figure 3: (Color online) (a) The invariant mass spectra of thermal di-electrons in full phase space. In the partonic phase, the main source is $q \bar{q} \rightarrow \gamma^{*} \rightarrow e^{+} e^{-}$(magenta-longdashed line). In the hadronic phase, the total contribution is the blue-dashed line, where the contribution from the $\rho$ meson (red-dotted line) dominates, and those from the $\omega$ and $\phi$ mesons are shown in magenta-short-dashed and brown-dashdotted lines respectively. (b) The invariant mass spectra of di-electrons for vector mesons $\rho, \omega$ and $\phi$ at the freezeout.

meson is given by

$$
\frac{d N_{l \bar{l}}^{f o}}{d^{4} p}=\frac{\alpha}{3}\left(\frac{e}{g}\right)^{2} \frac{m_{V}}{\Gamma_{V}} \frac{d N_{V}^{f o}}{d^{4} p} .
$$

where $\Gamma_{V}$ is the total decay width of the vector mesons. The vector meson momentum spectra at thermal freezeout can be expressed by the extended Cooper-Frye formula [5]

$$
\frac{d N_{V}^{f o}}{d^{4} p}=\frac{g_{s}^{\rho}}{4 \pi^{4}} \int_{T_{f}} d \Sigma_{\mu} p^{\mu} \operatorname{Im} D_{V} n_{B}(p \cdot u) .
$$

Since the lifetimes of $\omega$ and $\phi$ are much longer than the time scale of the freezeout process, we treat these contributions as in vacuum and neglect the medium effect. The imaginary parts of the $\omega$ and $\phi$ propagators can be given by the Breit-Wigner formula,

$$
\operatorname{Im} D_{\omega, \phi}^{f o}=-\frac{m_{V} \Gamma_{V}}{\left(M^{2}-m_{V}^{2}\right)^{2}+m_{V}^{2} \Gamma_{V}^{2}} .
$$

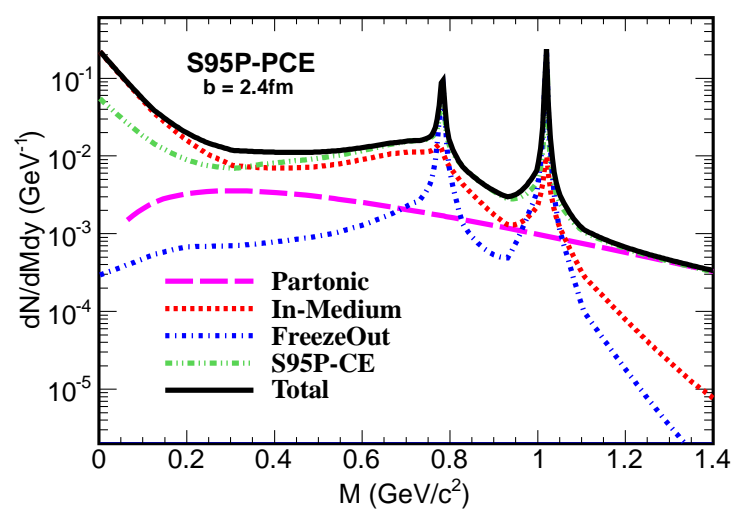

Figure 4: (Color online) The cocktail mixture of the partonic, in-medium and freezeout hadronic sources for di-electrons. The partonic, in-medium and freezeout hadronic contributions are in magenta-long-dashed, red-dotted, and blue-dashdotted lines, respectively. The total contribution is in the black-solid line.

But most of the $\rho$ mesons decay in the medium due to its short lifetime, so we include the medium effect in the $\rho$ meson propagator. In Fig.(3)(b)) are shown the invariant mass spectra of di-electrons from the freezeout vector mesons, where the sharp peaks of the $\omega$ and $\phi$ mesons can be seen compared to a much broader bump of the $\rho$ meson.

In Fig. (4) we sum over all sources we have considered. The full mass spectra have two sharp peaks of the $\omega$ and $\phi$ mesons at the freezeout same as in vacuum due to their long lifetime. For comparison the in-medium spectra are also shown where only a much lower peak from the $\phi$ meson is visible, indicating clear medium effects. Subtracting these sharp peaks of $\omega$ and $\phi$, the broadened spectrum of the in-medium $\rho$ meson can be seen. The partonic contribution dominates over the hadronic one when $M>1.1 \mathrm{GeV} / c^{2}$. These continuum-like IMR dileptons may provide a direct probe to the deconfinement phase transition in high energy heavy ion collisions. The different EOS give the similar structure but slightly different magnitude. It seems that the low mass enhancement favors the PCE scenario, we will come back to this point with details later in the following section.

\section{COMPARISON WITH DATA}

Different from the SPS energy, the charm quarks have a considerable production rate at the RHIC energy. So there is a large background from semi-leptonic decays of the charm hadrons. In this section we will estimate this background and compare our dilepton results with the data.

We use the event generator PYTHIA [34] (version 6.416 with CTEQ5L PDF) to simulate the background 

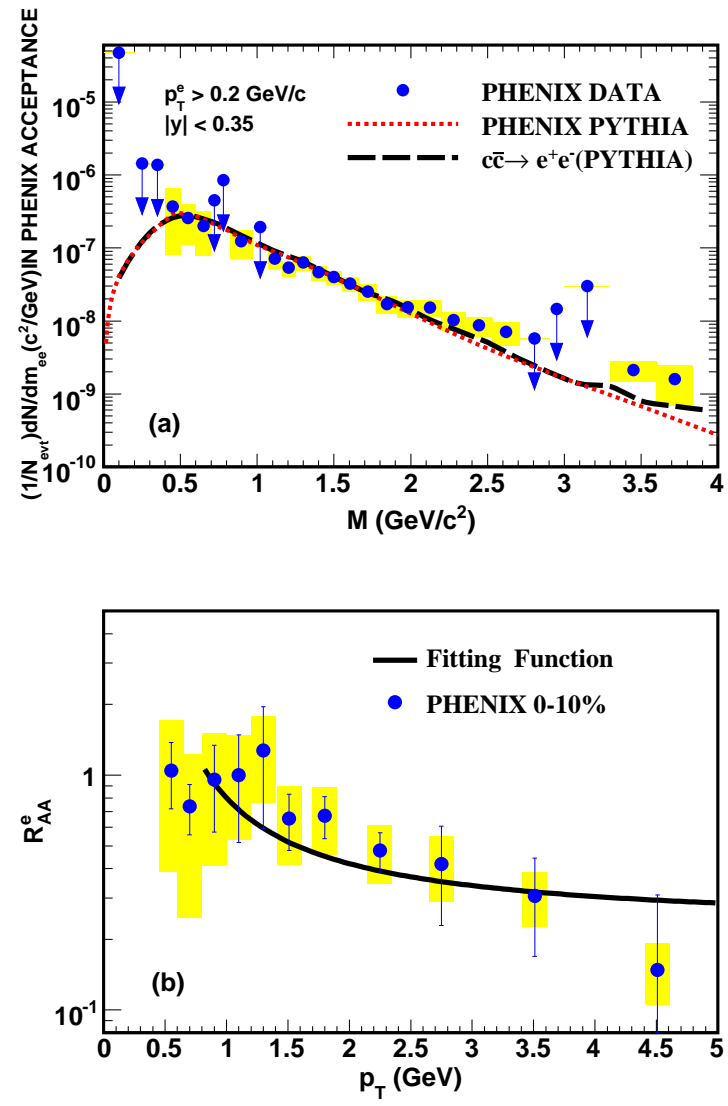

Figure 5: (Color online) The semi-leptonic decays of charm hadrons. (a) The re-scaled di-electron cross section from charm hadrons of semi-leptonic decays in $\mathrm{p}+\mathrm{p}$ collisions by PYTHIA. The data are taken from the PHENIX collaboration [14]. (b) The nuclear modification factor for nonphotonic electrons in central $\mathrm{Au}+\mathrm{Au}$ collisions from the PHENIX collaboration [36]. The fitting function is given in Eq. (8).

from semi-leptonic decays of the charm hadrons $\left(D^{0}, D^{ \pm}\right.$, $D_{s}$ and $\Lambda_{c}$ ). The PHENIX collaboration also tuned the parameters of PYTHIA [35] to fit the charm hadron data at SPS and FNAL and single electron data at ISR. The parameter dependences such as intrinsic $k_{T}$ and the parton distribution functions are also addressed in Ref. [14]. In our paper, we do not consider the fluctuations from these parameter.

In $\mathrm{p}+\mathrm{p}$ collisions, the dilepton yield in the mass range $[1.1,2.5] \mathrm{GeV} / c^{2}$ is dominated by semi-leptonic decays of charm hadrons. In the PHENIX acceptance the integrated yield of di-electrons per event from heavy-flavor decays in that range is $(4.21 \pm 0.28 \pm 1.02) \times 10^{-8}$ [14]. With the branch ratio for charm quarks to electrons [37] and the correction for the geometrical acceptance, the rapidity density of $c \bar{c}$ pairs can be estimated [14]. We use the PYTHIA event generator with the PHENIX acceptance to reproduce the spectra from charm hadron contribution, see Fig. (5)(a)). It can be seen that our results from PYTHIA (black-dashed line) are consistent with those given by PHENIX (red-dotted line). We obtain the cross section of $c \bar{c}$ pairs $\sigma_{c \bar{c}}=0.5 \mathrm{mb}$.

For $\mathrm{Au}+\mathrm{Au}$ collisions, we use the renormalized cross section in pp collisions and scale it by the mean number of binary collisions. We choose $N_{\text {coll }}=950$ for most central collisions. The charm quarks are mostly generated in the pre-equilibrium stage. In medium the $p_{T}$ spectra of the charm quarks as well as the angular correlation of the $c \bar{c}$ pairs could be modified due to its interaction with the thermalized partrons. The medium modifications of heavy flavors have been widely studied in, e.g., Ref. 38, 39]. To include the medium modifications in a simple way, we parametrize the nuclear modification factor of the single electron in the form,

$$
R_{A A}^{e}\left(p_{T}\right)=\min \left[1.0, \exp \left(a / p_{T}+b\right)\right],
$$

where $a=1.23$ and $b=-1.51$, see Fig. (5) (b)) for the fitting function and experimental data. Note that we have neglected the $\Upsilon$ contribution here. To get a realistic $p_{T}$ distribution for electrons, we use the original $p_{T}$ spectra obtained by PYTHIA and multiply them with $R_{A A}^{e}\left(p_{T}\right)$. Using the Monte Carlo method, we sample the momentum spectra in accordance with the resulting $p_{T}$ spectra for electrons and positrons respectively. In each event we randomly choose from the sample the momenta of one electron and one positron and combine them to a di-electron pair, from which the invariant mass and total transverse momentum of the pair can be determined. The modified invariant mass spectra by $R_{A A}^{e}\left(p_{T}\right)$ are found to be narrower than without such a modification as shown in Fig. (6(a)).

With the STAR acceptance (transverse momentum $p_{T}>0.2 \mathrm{GeV} / \mathrm{c}$ and pseudorapidity $\left|\eta^{e}\right|<1$ for an individual electron, rapidity $\left|y^{e e}\right|<1$ for a pair of electrons) and $p_{T}$ resolution, we compute the di-electron spectra in most central collisions and compare them with the STAR preliminary data of 0-10\% centrality, see Fig. [6(a)). We also included the Dalitz decay channels for $\eta$ [40] and $\omega$ : $\eta \rightarrow e^{+} e^{-} \gamma$ and $\omega \rightarrow e^{+} e^{-} \pi^{0}$. The $\eta$ contribution can be easily deducted as a background in the experiment due to its very long lifetime (about $1.5 \times 10^{5} \mathrm{fm} / \mathrm{c}$ ), which leads to its decay outside the freezeout scope.

The cooktail sum including the in-medium $\rho$ mesons can roughly reproduce the di-electron spectra in the LMR, see Fig. [6(a)). In Fig. (6)(b)), we show the total di-electron spectra (thick lines), the contributions from the open charm (green-dashed line) and the in-medium $\rho$ (thin lines) in the range $M \in[0,1.2] \mathrm{GeV} / c^{2}$. We found that the $\rho$ meson contributions (thin black-solid line) are submerged under the open charm one. This indicates that the charm backgrounds play an important role in the dilepton spectra at the RHIC energy. As we discussed in Sect. (III) we can tune $T_{f}$ to a lower value (e.g. $90 \mathrm{MeV}$ in red-dashed line) to increase the contribution from the in-medium $\rho$ mesons. The cocktail sum (thick red-dashed line) with the in-medium $\rho$ contribution (thin red-dashed line) for $T_{f}=90 \mathrm{MeV}$ is also shown. This seems to give a better fit to the data. Though the lower $T_{f}$ gives larger 

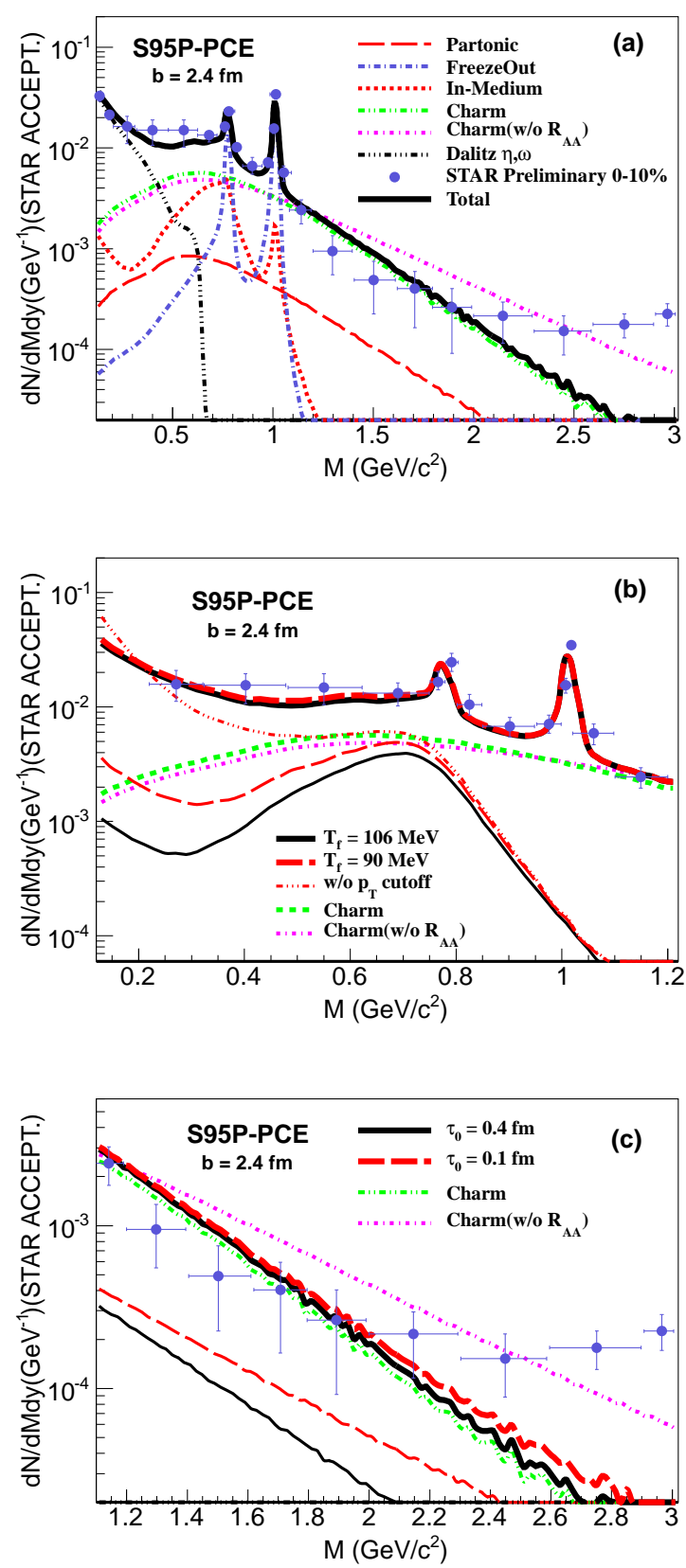

Figure 6: (Color online) The invariant mass spectra and the comparison with STAR preliminary data [17] in most central $(0-10 \%) \mathrm{Au}+\mathrm{Au}$ collisions with the STAR acceptance. The cocktail sums are in thick lines. See the text for detailed illustrations. (a) The results in $M=[0.2,3] \mathrm{GeV}$. (b) The results in $M=[0.2,1.2] \mathrm{GeV}$. The thin-balck/thin-redlong-dashed line denotes the contribution from the in-medium $\rho$ decays for $T_{f}=106 / 90 \mathrm{MeV}$. The thin-red-dashed-dotteddotted line denotes the contribution from the in-medium $\rho$ decays without the $p_{T}$ cutoff for $T_{f}=90 \mathrm{MeV}$. (c) The results in $M=[1.1,3] \mathrm{GeV}$. The thin-balck/thin-red-long-dashed line denotes the QGP contribution for $\tau_{0}=0.4 / 0.1 \mathrm{fm}$. broadenings of the $\rho$ spectra and low mass enhancements, the $\rho$ meson contribution is still smaller than the open charm one. This is because: (1) The nuclear modification factor enhances the charm contribution in the LMR; (2) Most of the low mass di-electrons from the in-medium $\rho$ mesons have low $p_{T}$, which are beyond the capability of the detectors and can not be measured. To support the point (2), we calculate the in-medium $\rho$ meson contribution incorporated by the STAR acceptance except the $p_{T}$ cutoff for electrons and positrons. The result is shown in the thin red-dashed-dotted-dotted line in Fig. (6)(b)). We can see a strong enhancement below the free $\rho$ mass.

With the nuclear modification factor for charm hadrons, we can roughly reproduce the di-electron spectra in the IMR, see Fig. (6)(c)). One can see that the thermal contributions from the QGP phase (thin blacksolid and red-dashed lines) are much smaller than the correlated charm decays (blue-dashed and green-dasheddotted-dotted lines). Now we try to look at if it is possible to increase the QGP thermal contributions in the IMR by the tuning parameters. We know that the thermal rate from the QGP is proportional to $T^{4} A \tau$ where $A$ is the transverse area [18]. To this end, in our model, we can tune the equilibration time and entropy density (initial energy density) with the constraint $s_{0} \tau_{0}=$ constant to keep the multiplicity rapidity density unchanged. The dilepton emission rates do not change much for different $\tau_{0}$. But for an earlier equilibration time, e.g. $\tau_{0}=0.1 \mathrm{fm}$ (thin red-dashed line), the partonic contribution is enhanced in the IMR, since the early equilibration time gives larger space-time volume of high temperatures, whose di-electron emissions mostly contribute to the IMR. But there is still a large gap between the contributions from charm hadrons and from the QGP. In addition, to lower the transition temperature will increase the space-time volume of the QGP phase and then dilepton rates from thermal partons. But this enhancement is almost in the LMR and will not significantly influence the IMR. So it seems that it is very difficult to extract the thermal sources from the backgrounds from charm hadron decays in the invariant mass spectra alone. Additional observables such as $p_{T}$ spectra and collective flows [18, 41] are also needed.

We show in Fig. (7) the results with the PHENIX acceptance 16. We see that the charm backgrounds still out-perform the in-medium $\rho$. The acceptance geometry pushes the charm hadron contributions toward the LMR. Using our cocktail sources, there is still a large unexpected excess of di-electrons in the LMR as reported by the PHENIX collaboration.

\section{SUMMARY AND CONCLUSION}

We investigate the di-electron low and intermediate mass spectra from the vector and charm hadrons in most central heavy ion collisions at ultra-relativistic energies. 


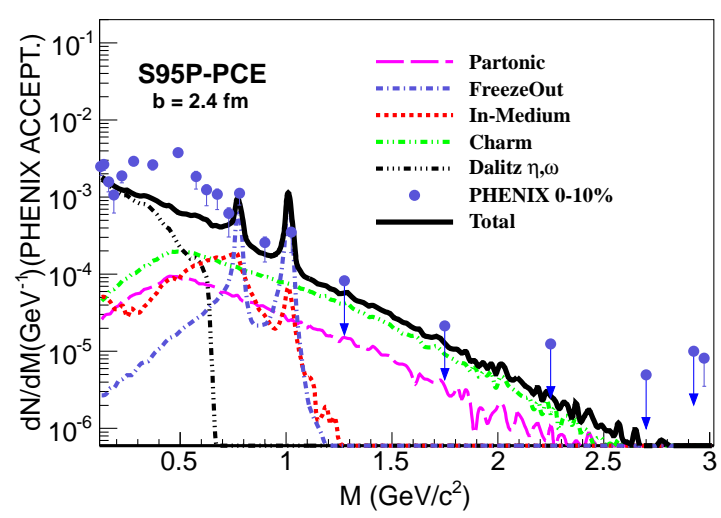

Figure 7: (Color online) Comparison with PHENIX data 16] in most central (0-10\%) $\mathrm{Au}+\mathrm{Au}$ collisions with the PHENIX acceptance.

The space-time history of the fireball is provided by a $2+1$ dimension ideal hydrodynamic model, whose parameters are fixed by fitting the transverse momentum spectra of long-life hadrons, i.e., pions, kaons and protons. Two types of equations of state are used. The medium effects of vector mesons from scatterings of vector mesons by mesons and baryons in the medium are considered. The di-electron emissions from in-medium vector meson decays can be evaluated via the imaginary parts of the vector meson propagators which are functions of spacetime through the temperature. Due to their longer lives than the time scale of the freezeout process, most of the $\omega$ and $\phi$ mesons may decay at the thermal freezeout, giving two sharp peaks in di-electron mass spectra. The contribution from the charm hadrons is modeled by the PYTHIA simulation of the proton-proton collisions and modified by the binary collision number and the nuclear modification factor for electrons.

The cocktail sum over all above sources and the partonic phase incorporated with the acceptances of the STAR detector is compared to the STAR preliminary data. The hadronic many body effective theory with a broadening rho meson spectral function can describe the STAR di-electron data in the LMR. With a parametrized nuclear modification factor for electrons from charm hadron decays, we can roughly reproduce the di-electron spectra in the IMR, though we still lack enough knowledge about open charm decays in medium, such as the modification from the dynamical correlation of $c \bar{c}$ pairs.

In conclusion, we find: (1) The detector acceptance especially the transverse momentum cutoff significantly suppresses the contribution from the in-medium $\rho$ meson in the mass region below the $\rho$ mass; (2) With the current set of parameters and detector acceptances, the backgrounds from charm hadrons dominate in the low and intermediate mass regions. Therefore it is impossible to extract the thermal sources of dileptons with the invariant mass spectra alone if the backgrounds from charm hadrons are not removed. Other observables such as transverse momenta and collective flows may provide additional tools to tag these sources. Future STAR programs such as the Heavy Flavor Tracker [42] and the Muon Telescope Detecctor are expected to improve the capability of identifying the backgrounds from charm decays and extracting the thermal souces.

Acknowledgement: QW is supported in part by the National Natural Science Foundation of China (NSFC) with grant No. 10735040. YFZ is supported in part by the National Natural Science Foundation of China (NSFC) with grant No. 10805046.
[1] L. D. McLerran and T. Toimela, Phys. Rev. D31, 545 (1985).

[2] K. Kajantie, J. I. Kapusta, L. D. McLerran, and A. Mekjian, Phys. Rev. D34, 2746 (1986).

[3] R. Rapp and J. Wambach, Adv. Nucl. Phys. 25, 1 (2000).

[4] J. Alam, S. Sarkar, P. Roy, T. Hatsuda, and B. Sinha, Annals Phys. 286, 159 (2001).

[5] H. van Hees and R. Rapp, Nucl. Phys. A806, 339 (2008).

[6] R. Chatterjee, D. K. Srivastava, U. W. Heinz, and C. Gale, Phys. Rev. C75, 054909 (2007).

[7] K. Dusling and S. Lin, Nucl. Phys. A809, 246 (2008).

[8] R. D. Pisarski, Phys. Lett. B110, 155 (1982).

[9] A. T. Martell and P. J. Ellis, Phys. Rev. C69, 065206 (2004).

[10] R. Arnaldi et al. (NA60), Phys. Rev. Lett. 96, 162302 (2006).

[11] H. van Hees and R. Rapp, Phys. Rev. Lett. 97, 102301 (2006).

[12] J. Ruppert, C. Gale, T. Renk, P. Lichard, and J. I. Kapusta, Phys. Rev. Lett. 100, 162301 (2008).
[13] S. Ghosh, S. Sarkar, and J.-e. Alam, Eur. Phys. J. C71, 1760 (2011).

[14] A. Adare et al. (PHENIX), Phys. Lett. B670, 313 (2009).

[15] K. Dusling, D. Teaney, and I. Zahed, Phys. Rev. C75, 024908 (2007).

[16] A. Adare et al. (PHENIX), Phys. Rev. C81, 034911 (2010).

[17] J. Zhao (STAR), J.Phys.G G38, 124134 (2011).

[18] J. Deng, Q. Wang, N. Xu, and P. Zhuang, Phys.Lett. B701, 581 (2011).

[19] T. Hirano and K. Tsuda, Phys.Rev. C66, 054905 (2002).

[20] P. Huovinen and P. Petreczky, Nucl. Phys. A837, 26 (2010).

[21] A. Bazavov et al., Phys. Rev. D80, 014504 (2009).

[22] C. Shen, U. Heinz, P. Huovinen, and H. Song, Phys. Rev. C82, 054904 (2010).

[23] F. Cooper and G. Frye, Phys. Rev. D10, 186 (1974).

[24] P. F. Kolb, J. Sollfrank, and U. W. Heinz, Phys.Rev. C62, 054909 (2000).

[25] B. I. Abelev et al. (STAR), Phys. Rev. C79, 034909 
(2009).

[26] S. S. Adler et al. (PHENIX), Phys. Rev. C69, 034910 (2004).

[27] J. Adams et al. (STAR), Phys. Rev. Lett. 92, 092301 (2004).

[28] B. I. Abelev et al. (STAR), Phys. Rev. C79, 064903 (2009).

[29] R. Rapp, Phys. Rev. C63, 054907 (2001).

[30] R. Rapp, C66, 017901 (2002).

[31] V. L. Eletsky, M. Belkacem, P. J. Ellis, and J. I. Kapusta, Phys. Rev. C64, 035202 (2001).

[32] R. Rapp, J. Phys. G34, S405 (2007).

[33] L. Alvarez-Ruso and V. Koch, Phys. Rev. C65, 054901 (2002).

[34] T. Sjostrand et al., Comput. Phys. Commun. 135, 238 (2001).

[35] K. Adcox et al. (PHENIX), Phys. Rev. Lett. 88, 192303
(2002)

[36] B. I. Abelev et al. (STAR), Phys. Rev. Lett. 98, 192301 (2007).

[37] S. Eidelman et al. (Particle Data Group), Phys. Lett. B592, 1 (2004).

[38] M. He, R. J. Fries, and R. Rapp (2011), 1106.6006.

[39] S. Ghosh, S. K. Das, S. Sarkar, and J.-e. Alam, Phys.Rev. D84, 011503 (2011).

[40] N. M. Kroll and W. Wada, Phys. Rev. 98, 1355 (1955).

[41] T. Renk and J. Ruppert, Phys. Rev. C77, 024907 (2008).

[42] Z. Xu et al., J. Phys. G32, S571 (2006).

[43] The EOS tables (by P. Huovinen), their analytic parametrizations (by T. Riley and C. Shen) and the list of included hadrons are available at the URL [https://wiki.bnl.gov/hhic/index.php/Lattice_calculatons _of_Equation_of_State]. 